\title{
Metamaterials with Giant Optical Activity
}

\author{
V. A. Fedotov, E. Plum, A. S. Schwanecke and N. I. Zheludev \\ Optoelectronics Research Centre, University of Southampton, SO17 1BJ, UK \\ Email: eplum@soton.ac.uk,web: http://www.nanophotonics.org.uk/niz/
}

\author{
Y. Chen \\ Central Microstructure Facility, Rutherford Appleton Laboratory, Oxfordshire, OX11 OQX, UK
}

\begin{abstract}
We demonstrate a novel type of chiral photonic metamaterial based on pairs of physically separated mutually twisted planar metal patterns. It exhibits very strong gyrotropy $\left(2500^{\circ} / \mathrm{mm}\right)$ in the visible, near-infrared and microwave spectral ranges.
\end{abstract}

Here, for the first time, we demonstrate a new type of widely scalable chiral photonic metamaterial, which exhibits strong gyrotropy in both the optical and microwave parts of the spectrum. It is a periodic bilayered structure, which is formed by an array of pairs of mutually twisted identical planar metal patterns located in parallel planes and separated by a dielectric layer.

Materials exhibiting strong gyrotropy were always of interest for various optoelectronic, life science microscopy and display applications but recently have started to attract a lot of attention as potential candidates for achieving negative refection. However, gyrotropic response of most of the naturally available materials is rather weak and therefore metamaterials have to be considered. From the metamaterial perspective it is very desirable that gyrotropy can be achieved by planar patterning using well-established planar nanotechnologies, which will make fabrication of optically active nano-structures a practical proposition. It has been suggested and recently demonstrated in the microwave part of the spectrum that electromagnetic coupling between layers of mutually twisted metal patterns can create 3D chirality showing strong gyrotropic response $[1,2]$.

The material for microwaves (see Fig. 1a) is made of copper rosettes edged on both sides of a standard PCboard with a pitch of $15 \mathrm{~mm}$. For the optical part of the spectrum the material is made of pairs of $\mathrm{Al}$ rosettes arranged in a grid with a pitch of $700 \mathrm{~nm}$ (see Fig. 2a). In both cases rosettes are twisted by $15^{\circ}$.

Fig. 1b shows spectral dependence of polarization plane rotation exhibited by the microwave chiral metamaterial between 4 and $8 \mathrm{GHz}$. In this frequency range the gyrotropy of the structure is resonant and exceptionally strong exceeding $40^{\circ}$ of polarization rotation per thickness of only $1 / 30$ of the wavelength.

The optical metamaterial exhibits very large optical activity in both visible and near IR spectral range (see Table 1), which is exceptionally strong near its transmission resonance (see Fig. 2b), namely at $1310 \mathrm{~nm}$, where polarization rotation reaches $2500 \% \mathrm{~mm}$.
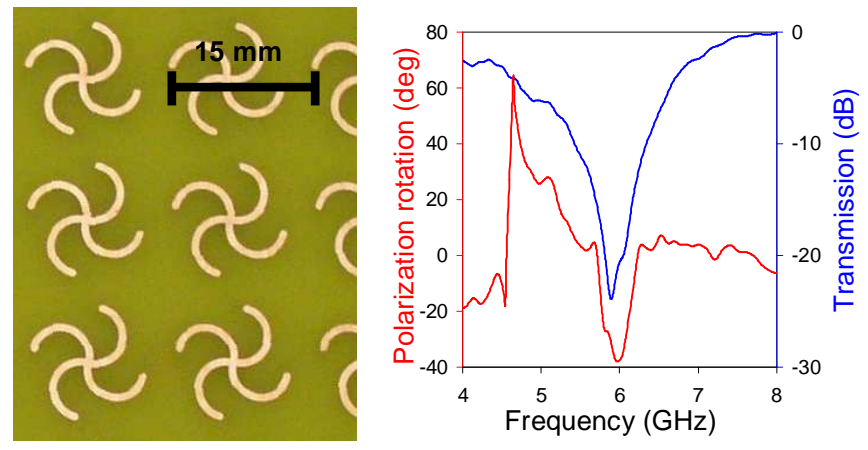

Fig. 1 (a) Microwave gyrotropic metamaterial consisting of twisted pairs of copper rosettes edged on both sides of 1.5 $\mathrm{mm}$ thick PC-board. (b) Rotation of the plane of polarization and transmission spectra by the microwavemetamaterial.
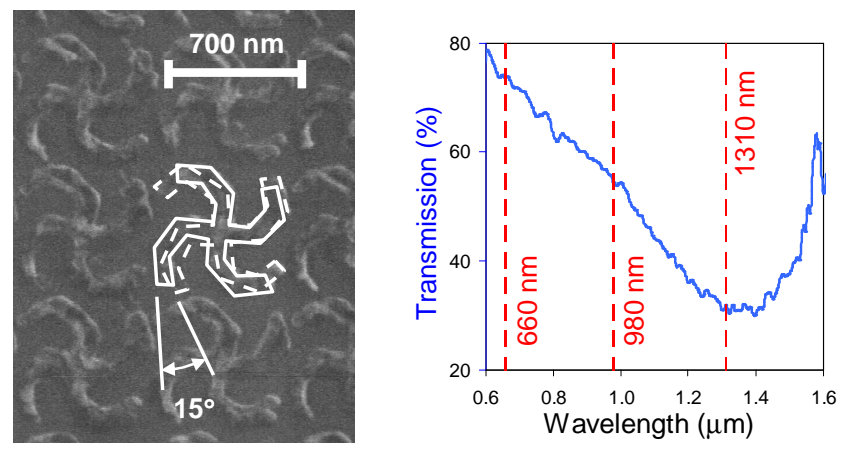

Fig. 2 (a) SEM micrograph of the gyrotropic metamaterial for the optical part of the spectrum, which consists of twisted pairs of $50 \mathrm{~nm}$ thick Al rosettes separated by only $50 \mathrm{~nm}$ of dielectric layer. (b) Transmission resonance of the twisted rosettes nano-structure. Red dashed lines show the wavelengths for which the polarization rotation has been measured.

\begin{tabular}{|l|l|l|l|}
\hline Wavelength [nm] & 660 & 980 & 1310 \\
\hline Optical Activity [ $\% \mathrm{~mm}]$ & 600 & 670 & 2500 \\
\hline
\end{tabular}

Table 1 Specific rotatory power of the optical metamaterial.

\section{References}

1. Y. Svirko, N. Zheludev, and M. Osipov, Appl. Phys. Lett. 78, 498 (2001).

2. A. V. Rogacheva, V. A. Fedotov, A. S. Schwanecke, and N. I. Zheludev, Phys. Rev. Lett. 97, 177401 (2006). 